\title{
Cue Awareness in Avoiding Effortful Control
}

Timothy L. Dunn ${ }^{1}$

Connor Gaspar ${ }^{2}$

Evan F. Risko ${ }^{2}$

1. Leeds School of Business, University of Colorado Boulder

2. Department of Psychology, University of Waterloo

All data, code, and pre-registration protocol are freely available via the Open Science Framework at osf.io/khtn3

This work was supported by a Discovery Grant from the Natural Sciences and

Engineering Research Council of Canada (NSERC) and funding from the Canada

Research Chairs program to E.F.R.

Address correspondence to: Timothy L. Dunn (tim.dunn@colorado.edu), Leeds Business School, University of Colorado Boulder, 995 Regent Dr. Koelbel Building, 419 UCB, Boulder, CO 80309

Word count: 9517 


\begin{abstract}
Based on a cue-based metacognitive account, cognitive effort is the result of an inferential evaluation made over explicitly available cues. Following from this account, we present here a pre-registered experiment that tested the specific hypothesis that explicit awareness of cues that are aligned with cognitive demand is a prerequisite in avoiding effortful lines of action. We attempted to modulate levels of effort avoidance behavior by introducing an incentive (between-subjects) to monitor two lines of action that, unbeknownst to individuals, varied in the probability of a task switch. Importantly, previous research has demonstrated that the difference in these probabilities is relatively opaque to individuals. We did not find strong evidence for our incentive manipulation having an effect on demand avoidance as indexed by individuals' choices in a block of the task where avoiding effort was instructed. However, when considering awareness generally, we do find that being aware of the task-switching cue appears to increase the likelihood of demand avoidance. We consider these results within the context of the metacognition of cognitive effort.
\end{abstract}

Keywords: cognitive effort, conscious awareness, metacognition, cognitive control, cue-utilization 


\section{Cue Awareness in Avoiding Effortful Control}

Generally, it is hypothesized that engaging the control systems that are required to override a habitual or automatic response is effortful, and the putative level of effort involved in such engagement weighs as a cost to be avoided in decision-making (Botvinick, 2007; Botvinick \& Braver, 2015; Inzlicht, Schmeichel, Macrae, 2013; Kool, McGuire, Rosen, \& Botvinick, 2010; Kool, McGuire, Wang, \& Botvinick, 2013; Kool \& Botvinick, 2014; Kurzban, Duckworth, Kable, \& Myers, 2013; Shenhav, Botvinick, Cohen, 2013; Shenhav et al., 2017; Westbrook \& Braver, 2015; 2016). Such controllinked behaviors can range in granularity from attempting to suppress inappropriate responses to specific stimuli (e.g., the flankers task, Eriksen \& Eriksen, 1974; the Stroop task, see MacLeod, 1991 for a review), to switching between tasks (Monsell, 2003), to engaging in deliberate analytical thinking rather than automatic intuitive thinking (Pennycook, Fugelsang, \& Koehler, 2015), to resisting temptations, regulating emotions, and reaching novel planned goals (see de Ridder et al., 2012 for a review). Here, we provide a pre-registered test of a recent account that proposes that awareness of cues (associated with cognitive demand) is a necessary condition in avoiding lines of action associated with heightened effortful control.

\section{The Relation Between Awareness and Cognitive Processing}

How individuals evaluate and avoid effortful lines of action has recently received much attention (e.g., Botvinick, Huffstetler, \& McGuire, 2009; Gold et al., 2014; Desender, Van Opstal, \& Van den Bussche, 2017; Dunn, Lutes, \& Risko, 2016; Kool et al., 2010; McGuire \& Botvinick, 2010; Risko \& Gilbert, 2016; Westbrook, Kester, \& Braver, 2013; Westbrook \& Braver, 2015). Manipulations of cognitive effort have most 
commonly focused on manipulating the level of controlled, relative to automatic, processing required. That is, the increased engagement of the neural systems associated with controlled processing systems (i.e., the system spanning the dorsolateral prefrontal cortex, the anterior cingulate cortex, and the intraparietal cortex) has been argued to be intimately linked to the "cost" (i.e., the aversive component) associated with effort that individuals may seek to avoid in the absence of an offsetting reward (for a review see Botvinick \& Braver, 2015). Under some specific frameworks, cognitive effort, as a limited capacity, registers as a cost insofar as it is recruited in response to varying control requirements (e.g., Shenhav et al., 2017).

Recently, the relation between effort-based decision-making and conscious awareness has received attention. This relation entails specifically considering whether conscious awareness of cognitive effort is a prerequisite to individuals generating behaviors (i.e., decisions or evaluations) that are in line with the notion of effort minimization (Desender et al., 2017; Dunn et al. 2016; Mulert, Menzinger, Leicht, Pogarell, \& Hegerl, 2005; Naccache et al., 2005; Shenhav et al., 2017; Westbrook \& Braver, 2015). Two straightforward competing hypotheses can be derived from this consideration related to effort-based decision-making: (1) effort avoidance can proceed at the implicit level (i.e., without conscious awareness), or (2) effort avoidance can only occur when individuals become explicitly aware of attributes of a task that signal a difference in demand, or generate a feeling of conscious effort. Importantly, the former does not preclude the influence of explicit awareness in action selection; rather, both likely influence behaviors. In contrast, the latter does preclude the influence of effort avoidance proceeding as a wholly implicit process. Below, we briefly review the 
available evidence related to both hypotheses in relation to cognition and control broadly (for a more thorough review see Desender \& Van den Bussche, 2012), and cognitive effort specifically.

\section{Cognition and Behavior in the Absence and Presence of Awareness}

Implicit learning has long served as an example of cognition without awareness (for a review see Dienes \& Perner, 1999). Reber (1989) defines implicit learning as, “...the process by which knowledge about the rule-governed complexities of the stimulus environment is acquired independently of conscious attempts to do so" (p. 219). Perhaps the most pervasive paradigm used to study implicit learning is artificial grammar tasks. Here, participants are asked to memorize grammatical strings of letters generated by a finite-state grammar, informed of the existence of the complex set of rules that constrains letter order, and are finally asked to classify grammatical and non-grammatical strings (Reber 1967; 1989). The general finding is that individuals are able to correctly categorize letter strings at levels above chance even while lacking awareness of what the grammatical set of rules were (e.g., Dienes \& Altman, 1997; Dienes, Altman, Kwan, \& Goode, 1995; Dienes, Broadbent, \& Berry, 1991; Reber 1967; 1976; Reber, Allen, \& Regan, 1985; Reber \& Lewis, 1977). This kind of implicit learning can also play a role in strategy selection, a form of behavioral control. Payne and colleagues (1993, p. 201) noted that individuals can likely configure their behaviors in an adaptive manner even though they may be unable to report specific factors of a task that would be expected to drive strategy selection. Indeed, Reder and Schunn (1996; see also Reder 1988) argue that participants will continue to use learned beneficial strategies (in terms of performance) 
without explicit awareness that this behavior is taking place. Thus, individuals seem able to use information not available to conscious report to control their behavior.

Engagement of controlled processing systems has been long associated with increases in cognitive effort (Botvinick \& Braver, 2015). Interestingly, cognitive control also seems able to be engaged without conscious awareness. Much of this work has focused on conflict adaptation, which consists of preventing irrelevant information from exerting a detrimental influence on ongoing performance (e.g., the Gratton effect; Gratton, Coles, \& Donchin, 1992). For instance, if individuals learn to withhold a response based on a specific cue, their responses will similarly slow down when the cue is presented unconsciously through masking. Though unconscious, processing of these cues was correlated with areas commonly involved in inhibitory control including the inferior frontal cortex (IFC) and the pre-supplementary motor area (pre-SMA; van Gaal, Ridderinkhof, Scholte, \& Lamme, 2010). In addition, Diede and Bugg (2017) demonstrated response adaption effects, as indexed by response times and a difference in average pupil diameter (i.e., a common index of cognitive demand; see, Joshi, Li, Kalwani, \& Gold, 2016) across different conflict frequency conditions, despite the fact that individuals were unable to report differences across conditions in conflict frequency (see also, Bugg \& Diede, 2017 for an example utilizing a pre-cued list paradigm).

Though control without awareness has been widely demonstrated, several have argued the contrary. Dehaene and Naccache (2001) suggest that without reaching consciousness, stimuli are unable to initiate general top-down control. Here, consciousness holds a special position in initiating planning, evaluation, and control. Following from this notion, Desender and colleagues (2014) demonstrated that conflict 
adaptation effects were only triggered by a subjective experience of conflict, and this experience was dissociated from the actual observed congruency effects (see also, Desender, van Lierde, \& van den Bussche, 2013; Reuss, Desender, Kiesel, \& Kunde, 2014; Questienne, Van Opstal, Van Dijck, \& Gevers, 2016; c.f., Abrahamse \& Braem, 2015). In a similar setting, Heinemann, Kunde, and Kiesel (2009; see also Schouppe, de Ferrerre, Van Opstal, Braem, \& Notebaert, 2014) examined the role of awareness in generating the context-specific proportion congruent (CSPC) effect. The CSPC effect is the demonstration that congruency effects can vary depending on context-features that appear more or less simultaneously with the response stimuli (Crump, Gong, \& Milliken, 2006). Heinemann and colleagues (2009) used both weakly and strongly masked irrelevant prime information (i.e., the distractor) in a Flanker task. The context manipulation did not produce the specific CSPC effects when the prime was strongly masked. It was thus concluded that conscious access to the incompatible prime was necessary for the effect to unfold. Thus, as noted by Desender and colleagues (2014), “...participants will adapt their behavior only if they have an experience of conflict" (p. $680)$.

Thus far, we have briefly reviewed several contexts in which cognition and behavior can be influenced by implicit and explicit processes. Focusing on control, the evidence suggests that awareness can play a role in some contexts, but does not seem to be required to initiate controlled processes. This is of particular interest for the current investigation given controlled processes' relation to cognitive effort. Next, we turn to the available (albeit limited) evidence specifically focused on examinations of cognitive effort and awareness. 


\section{Cognitive Effort and Awareness}

In their review of cognitive effort Westbrook and Braver (2015) noted that the course of control costs (specifically opportunity costs) need not always be conscious to influence behavior. However, as discussed by Hagger (2013), the opportunity cost model of effort proposed by Kurzban and colleagues (2013) is arguably unclear on whether the processes associated with computing these costs work at an automatic-level of processing outside of conscious awareness. Hagger (2013) notes that utilizing "next-best alternatives" in computations may imply a need for conscious awareness of what those alternatives are. Nonetheless, there is some empirical evidence to support the claim that effort avoidance can unfold in the absence of awareness of effort cues. In their influential work on cognitive demand avoidance, Kool and colleagues (2010) utilized a demand selection task (DST) where individuals made free choices about which of two options they preferred to engaged in on a trial-by-trial basis. Critically, and unbeknownst to participants, these options were manipulated to vary in the levels of cognitive demand (e.g., more switching vs. less switching). Interestingly, in the first of their experiments, Kool and colleagues (2010) demonstrated that eight of their 43 participants produced patterns of choices consistent with effort avoidance, even though they self-reported no awareness of the difference of the probability of a task switch across the two choice options. Furthermore, Botvinick (2007) notes additional demonstrations of behavior consistent with effort avoidance for subsets of individuals who lack explicit awareness of differences in cognitive demand.

The specific association between cue awareness and cognitive effort avoidance has recently been highlighted within a cue-based metacognitive account of cognitive 
effort offered by Dunn and colleagues (2016; see also Dunn \& Risko, submitted).

Following from cue-utilization accounts of metacognitive judgments (e.g., Koriat, 1997;

Mueller, Dunlosky, \& Tauber, 2016), they suggested that cognitive effort results from an inferential metacognitive evaluation made over explicitly available cues. Within the study of metacognition, a distinction is often made between two sources of evaluation: (1) inferential processes, and (2) sheer subjective feelings. Importantly, the former entails a more conscious process, and the phenomenological quality of the latter lies in a variety of subtle processes that occur below full consciousness (Kelley \& Jacoby, 1996; Koriat, 2000; Koriat, Nussinson, Bless, \& Shaked, 2008).

Basic hierarchical metacognitive models posit a lack of direct-access between two cognitive systems. Specifically, evaluative meta-level processes which are associated with areas of the prefrontal cortex (PFC) imperfectly monitor information in the lower object-level of cognitive processing (e.g., task demand) which are associated with areas of the posterior cortex (Fleming \& Dolan, 2012; Nelson \& Narens 1990; Shimamura, $2000 ; 2008)$. Given the disconnect between these two systems, a cue-based metacognitive accounts posit that individuals monitor variation across lines of action by way of cues during action selection (e.g., effort avoidance). This is in contrast to having the ability to index and utilize some veridical signal associated with cognitive demand at the objectlevel. Following from this conception, then, individuals' judgments and behaviors related to effort would be expected to dissociate (but not always) from common measures of cognitive demand (e.g., performance, physiological measures). This finding has indeed been demonstrated across many contexts (Desender et al., 2017; Dunn et al., 2016; Dunn \& Risko, 2016; Dunn \& Risko, submitted; Kool et al., 2010; Gold et al., 2014; McGuire 
\& Botvinick, 2010; Naccache et al., 2005; Westbrook, Kester, \& Braver, 2013). Dunn and colleagues (2016) further suggested that an explicit awareness of cues may be required to avoid effortful lines of action given the decision is generated at the introspective meta-level of processing; a crucial aspect of consciousness (Cleeremans, Timmermans, \& Pasquali, 2007; Lau, 2008). A recent set of experiments directly supported this hypothesis.

Desender and colleagues (2017) had individuals freely choose between performing a task in either high-demand or low-demand conditions. Demand was manipulated by the proportion of response conflict trials associated with each option. Critically, subliminal priming was utilized to ensure that participants were not aware of the visual stimuli (i.e., congruency and incongruency) creating the difference in demand across options. That is, the cues from which effort could have been inferred were putatively unavailable to individuals. Accordingly, across three experiments, only individuals who became aware of these differences (using measures from signal detection theory) demonstrated choices consistent with demand avoidance. This finding held even in light of identical task performance across aware and unaware individuals. Moreover, a multiple regression confirmed that awareness was indeed the main factor driving demand avoidance. In line with the cue-based metacognitive account of effort (Dunn et al., 2016), the authors argued that actual differences in cognitive demand across options does not drive demand avoidance, but rather, it is the awareness of the cues related to demand that signal a difference across options that drives demand avoidance. 


\section{Present Investigation}

The present investigation sought to extend the hypothesis that awareness of effort cues is a prerequisite to evaluating and avoiding effortful lines of action. We made use of a recent demonstration of individuals' relative lack of awareness of differences in task switching probabilities. Dunn and Risko (submitted) showed that effort avoidance across two options associated with a $70 \%$ and $30 \%$ probability of task-switch respectively was at chance levels, and self-reported awareness of the specific task switching cue (i.e., more vs. less switching) was relatively low. This finding is unsurprising given many findings of individuals being unaware of even large proportion manipulations (e.g., Crump, Gong, \& Milliken, 2006; Risko \& Stolz, 2010; Schmidt, Crump, Cheesman, \& Besner, 2007). Here we looked to moderate levels of effort avoidance above chance by introducing an incentive to raise awareness of the switching cue without affecting performance in the DST (see Botvinick \& Rosen, 2009; Desender, Van Opstal, \& Van den Bussche, 2017; Dunn et al., 2016; Dunn \& Risko, submitted; Gold et al., 2014; Kool et al., 2010; McGuire \& Botvinick, 2010). Prior to outlining our specific predictions, a description of operational definitions is in line.

Presently, we test the cue-based metacognitive account of cognitive effort described above (Dunn et al., 2016; Dunn \& Risko, submitted). We use the term effort cue to refer to an attribute(s) of a task available to an agent on which an inference about demand could be made. Demand in the present context is associated with task-switching (i.e., continually configuring processing resources to achieve some goal; Monsell, Yeung, \& Azuma, 2000) where more switching (relative to less switching) can be argued to reflect higher demands on the control system (Kool et al., 2010) such as a need for a more 
proactive level of control (Braver, 2012). Here, the critical cue is the difference in the probability of a task-switch on a trial-by-trial basis (i.e., $30 \%$ vs 70\%) across tasks, where the presumption is that individuals hold the belief that more switching is more effortful than less switching (e.g., Kool et al., 2010). An evaluation of effort can be utilized whether situated in a task (e.g., disengaging from an action through a retrospective evaluation of effort) or not (e.g., avoiding an action outright through a prospective evaluation of effort; see also Westbrook \& Braver, 2015). We are concerned with the latter for the current investigation. An evaluation of cognitive effort does not necessarily have to track with cognitive demand (i.e., here, the demand associated with taskswitching), but can, if cues and the level of demand are aligned in the same direction (Dunn \& Risko, 2015). For example, in the present context the task switching cue (i.e., $30 \%$ vs. $70 \%$ ) is aligned in this positive direction with the level of demand. Nonetheless, from the above we posit that this difference in switching is generally opaque to individuals.

Last, we define awareness as the introspective awareness of effort cues while situated within a task. Here, we follow Block's (1995) distinction between phenomenaland access-consciousness. Importantly, we mean the latter as pertaining to awareness. A perceptual state or experience is access-conscious, if its content (e.g., a cue) becomes available to the higher-level cognitive processes whereby it can be used to control reasoning and behavior. Similarly, Snodgrass, Bernat, and Shevrin (2004) propose that reflective consciousness operates as a higher-order metacognitive process which selects subsets of phenomenal experience for further evaluative processes. Information becomes accessible when it is sufficiently clear to produce reasonable confidence and deemed 
task-relevant. One key element of this form of consciousness is reportability (Block, 1995; Overgaard \& Sandberg, 2012; Snodgrass, Kalaida, \& Winer, 2009). Positioned within the cue-based metacognitive account of effort, then, the cue or cues in question (here, the difference in the probability of task switching that are associated with cognitive demand) must reach this level of awareness for an individual to avoid the more effortful line of action.

In the current experiment individuals completed a variant of the DST consisting of three blocks: the first two were "forced-choice" where individuals garnered experience with both of the options, the third block was "free-choice" were individuals were specifically instructed to generate a less-effortful preference for one the options. As noted above, demand was manipulated by the probability of a task-switch on a trial-by-trial basis (i.e., $30 \%$ for the low-demand option and $70 \%$ for the high-demand option). In addition, we specifically indexed demand by performance in the first two blocks, first by overall performance (i.e., response times and errors) across the two options (i.e., the demand effect), and second in terms of the difference between repeat and switch trials (i.e., switch costs). Following from previous work using task-switching in the DST (e.g., Botvinick \& Rosen, 2009; Dunn \& Risko, submitted; Kool et al., 2010), we expected overall response times (RTs) to be longer for the high-demand option and switch costs to be smaller in the high-demand option. We do not expect errors to vary across options given previous work using the same probabilities of switching (Dunn \& Risko, submitted).

Previous work has demonstrated that performance-based incentives can enhance performance in task-switching contexts (Aarts et al., 2010) and predict greater neural 
activity in control related areas such as the PFC and ACC (Westbrook \& Braver, 2016).

Thus, in an attempt to leave performance unaffected across the incentive groups, we used an incentive that was not performance-based nor specific to the task switching manipulation (i.e., individuals would try harder when switching in the incentive condition). Specifically, the incentive manipulation consisted of an instruction prior to the DST that correctly answering a question about the task would be rewarded with a $\$ 2$ bonus. Following recent work (Dunn \& Risko, submitted), we hypothesized that individuals in the no-incentive condition will demonstrate choices in the third free-choice block near chance (i.e., no demand avoidance). We further hypothesized that individuals in the incentive condition will demonstrate rates of demand avoidance above chance following from the notion that the incentive should increase awareness of the taskswitching cue. We index awareness by a post-DST no-loss gambling task. In the no-loss gambling task, individuals were first asked whether one option switched more than the alternative. They then had the option to stick with their answer for the $\$ 2$ bonus or accept a 50/50 gamble for the chance to win the $\$ 2$ bonus (see below for more details). Thus, individuals made a judgment about their experience in the form of the switching question, and made a choice that reflects the strength of their experience (i.e., their confidence) in the form of gambling or not. No-loss gambling controls for risk-aversion given the individuals cannot lose any reward (i.e., the $\$ 2$ bonus offered presently), and provides a high motivation to respond in an unbiased manner given the direct contrast between staying (i.e., on their answer) or gambling. Importantly, this procedure provides a clear indication of guessing through the choice of a 50/50 gamble in a non-idiosyncratic way: when a participant chooses to gamble, they are betting on a random process rather their 
own answer (Dienes \& Seth, 2010; Schurger \& Sher, 2008). With regard to the no-loss gambling task, we hypothesize that individuals in the incentive condition will overall be more accurate and confident in their answer to the switching question given the predicted heightened awareness of the cue relative to the no incentive condition. Furthermore, those individuals accurately identify the high-switching option and are confident in their answer to the switching question should exhibit higher rates of demand avoidance relative to those individuals that do not become aware.

\section{Experiment 1}

\section{Method}

In the following we report how we determined our sample size, all data exclusions, all manipulations, and all measures in the study (Simmons, Nelson, \& Simonsohn, 2012). The pre-registration protocol and all data for the study is freely available at osf.io/khtn3.

\section{Participants}

Initial sample. Two hundred (171 females) University of Waterloo undergraduate students participated in the experiment in exchange for research credit. Optional stopping methods were utilized given that Bayes factors $(\mathrm{BF})$ are the primary means of inference for the current study (Rouder, 2014; Schönbrodt, Wagenmakers, Zehetleitner, \& Perugini, 2017). Initially, a starting sample size of $N=40$ was selected ( $n=20$ for each betweensubject group), after which data collection would terminate if a BF across the incentive and non-incentive groups for low-demand selections (see below) demonstrated evidence for either the null (i.e., no difference in low-demand selections) or alternative hypothesis (i.e., some difference in low-demand selections) using a BF threshold value of greater than or equal to 10 (i.e., strong evidence; Jeffreys, 1961; Lee \& Wagenmakers, 2013). If 
the BF computed for the difference between the low- and high-demand conditions did not reach this threshold, then an additional sample of 40 participants was collected. This was pre-determined to carry on until $N=200$, at which point data collection would be terminated regardless of the level of evidence reached.

Exclusions and rationale. Following collection of the $N=200$ sample, we required an additional 17 individuals due to an imbalance of two between-subject order variables. These variables were the left/right position of the high-demand deck and the presented order of high/low demand decks during the practice sessions. Replacement was done by identifying the most recently recruited individuals' whose data met the condition-overbalance criteria. Additionally, one individual was identified as not completing the first two forced-choice blocks as instructed, therefore their data was excluded from all analyses. Thus, our final sample size was $N=199$ (see Figure 1). 
Figure 1. Results from the Bayesian optional stopping procedure.

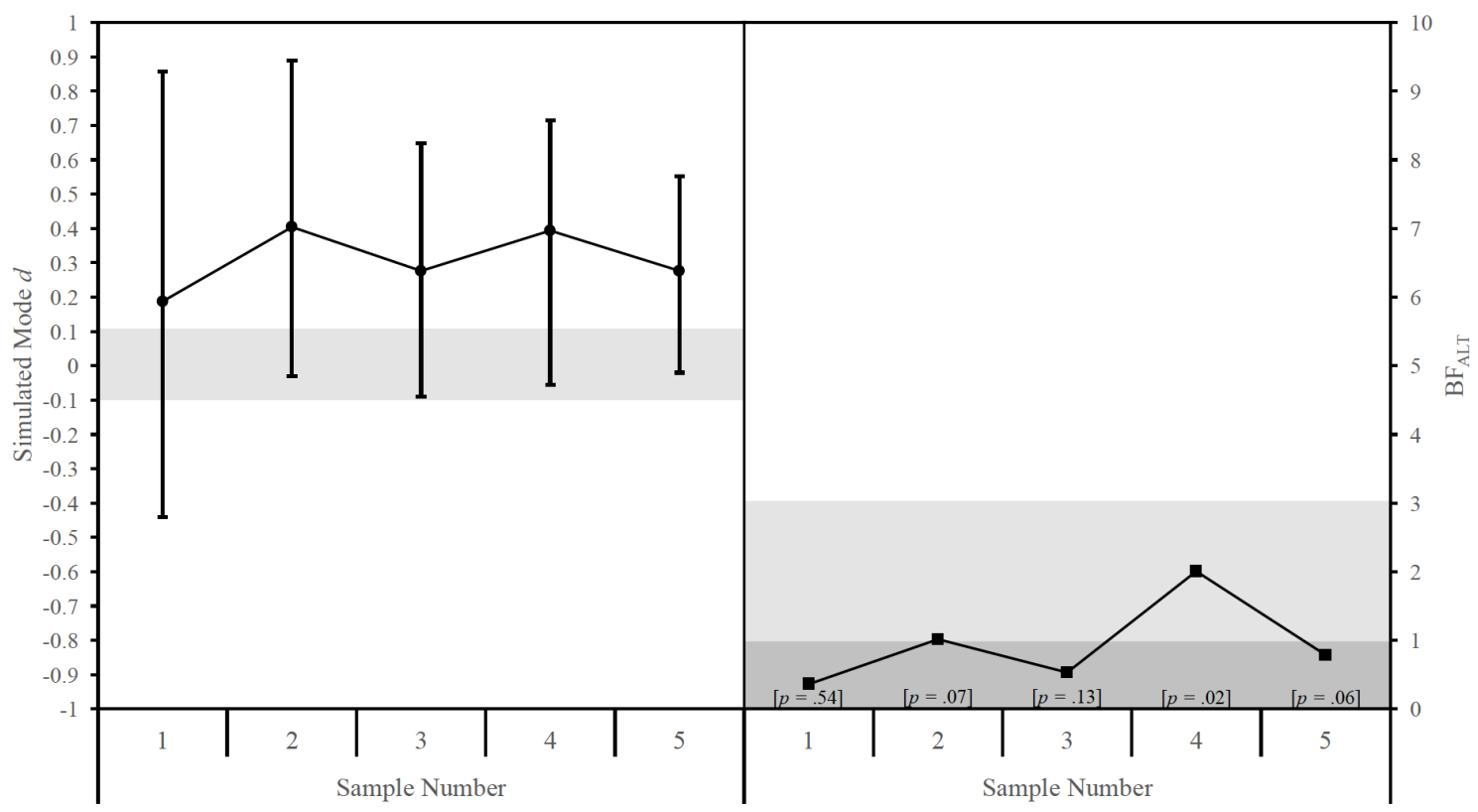

Note: all analyses are for the focal effect of incentive versus no incentive on the overall proportion of less-effortful choices for the low-demand option in the third block of the DST (i.e., a positive effect size). Each sample consisted of $n=20$ per group and the analyses shown here aggregate samples at each step (i.e., sample number five contains the entire final sample of $N=199$ ). The left panel displays Bayesian estimation analyses of the mode Cohen's $d$ value for the focal effect. Error bars represent 95\% Highest Density Intervals (HDI). The shaded band represents a Region of Practical Equivalence (ROPE) ranging from $d=-1$ to $d=1$ (Kruschke, 2014). The right panel displays the BFs for the between-subject tests for each sample number. The unshaded area represents the range of BFs associated with the "Moderate" evidence category, the lighter band represents the "Anecdotal" category, and the darker band represents the area in which a BF would represent evidence for the null hypothesis (i.e., no difference across the groups; Lee \& Wagenmakers, 2013). In addition, $p$-values for each between-subject $t$-test are presented in brackets.

\section{Design}

The present experiment utilized a 2 (Incentive: informed vs. uninformed) x 2 (Demand: high vs. low) mixed design. Incentive was manipulated between-subjects and demand was manipulated within-subjects. 


\section{Apparatus}

The DST (Kool et al., 2010) was programmed in MATLAB using the Psychophysics toolbox (Brainard, 1997; Pelli, 1997; Kleiner et al, 2007) ${ }^{1}$. The program was delivered using a 24" LCD monitor with participants positioned approximately $70 \mathrm{~cm}$ away. Participants utilized a wired standard optical mouse in order to respond in the DST.

\section{Stimuli}

Stimuli followed previous experiments utilizing the DST (see Dunn \& Risko, submitted; Gold et al. 2014; Kool et al., 2010). The decks presented were either low- or high-demand and presented to the left and right of center on the screen. The locations of the high- and low-demand options were not communicated to participants. The lowdemand option consisted of a $30 \%$ probability of a task-switch, whereas the high-demand option consisted of a $70 \%$ probability of a task switch. Once the cursor was placed on a target centered on the screen, both options were activated and participants were free to choose an option that would reveal a colored digit (blue or yellow). Digits consisted of "1", "4", "7", and "8". At this point, the participant was required to make a response. If the digit was blue, the participants were required to perform a magnitude judgment (greater than or less than 5). If the number was yellow, then participants were required to perform a parity judgment (odd or even). Participants clicked left on the mouse for a less than five or odd response, or right clicked for a greater than five or even response.

\section{Procedure}

Individuals first completed a practice session with the DST to ensure understanding of the instructions and task procedure. The practice session consisted of three blocks of trials. In the first block of trials, participants were asked to respond to 
numbers contingent on the color the number was presented in. All participants were provided with a reference graphic of the correct response mappings during the first practice block. After the first block, participants' access to the reference was removed and they were informed that they would receive explicit feedback about their accuracy in the next practice block. Participants were instructed that they were required to achieve an overall accuracy of $>=90 \%$ (at least $54 / 60$ trials correct) to proceed to the main experiment. If the accuracy threshold was not satisfied, individuals were allowed to retry the practice session. If they were unable to meet the threshold on the second try of their practice, the experiment was terminated due to time constraints. The third block of practice consisted of exposure and experience with the primary DST where participants were to select from one of two patches, and subsequently respond to a number similarly to the main experiment.

Upon completion of the practice session, individuals were given instructions on how to complete the main experiment. The main DST consisted of three blocks of 50 trials each. In the first two blocks, participants were instructed to select the cued circle, which would switch after 50 trials to ensure equal experience with each deck $^{2}$. In the final 50 trials, participants were asked to begin selecting the deck they believed to be least effortful (see Dunn \& Risko, submitted). At the end of the instructions, the incentive manipulation was delivered. Here, participants in the incentive group were informed that after the DST, providing a correct answer to a question about the task would reward them with a $\$ 2$ bonus in addition to earning a research credit. However, individuals in the noincentive condition were not provided this instruction and therefore had no knowledge of this incentive prior to completing the DST. Characteristics of the DST such as the order 
of the forced-choice blocks pertaining to low- and high-demand and the location of each option were randomized and counterbalanced across all participants.

At the conclusion of the main DST, all participants completed a no-loss gambling task (see Appendix A. for full protocol). Again, the no-incentive group was unaware of this task prior to the main DST. First, participants were instructed that they were to choose from one of two facedown cards that were labeled either "Win" or "No Win" (though both cards were labeled "Win" given everyone was paid the bonus). Choosing the "Win" card would result in winning a \$2 bonus whereas the "No Win" card would not. After selection, both cards remained facedown so that the participant would not know the outcome. Next, participants were asked to think back to the main DST, and whether the deck on the left or the right (counterbalanced) tended to switch between colors more often than the other deck (e.g., "In the previous part of the task the option on the left tended to switch between colors more often than the option on the right?"). Participants were informed that a correct answer to the question would lead to winning the $\$ 2$ bonus, whereas an incorrect answer would not. Last, participants were then asked to make a choice between either the card they selected prior to their answer about the task, or the answer they provided as to which event they would like to determine their winnings. Following this decision, all participants were provided with a $\$ 2$ regardless of their answers.

\section{Results}

Results are reported first for overall block performance (i.e., response times and accuracy), switch costs in terms of response time (RT) and accuracy derived from the first two forced-choice blocks (see Table 1), followed by the free-choice block, and no- 
loss gambling data. Bayes Factors (BF) were computed using the BayesFactor package (Morey \& Rouder, 2015) in R (R Core Team, 2014). With respect to the calculation of Bayes factors for ANOVA's and proportion analyses, a default fixed r scale $=1 / 2$ was utilized. Bayes Factors calculated for the ANOVA models by default are presented as referenced to the random effect error model as the null model. We present these BFs first in the sections below. We additionally focus on specific comparisons between high-level models using BFs where appropriate (e.g., the interaction model versus the main effect model). A default prior of $r=\sqrt{2} / 2$ (i.e., "Medium") was utilized for analyzing differences in means for the demand selection data. Grades of evidence categories for BFs follow the criteria outlined by Lee and Wagenmakers (2013; see also Jeffreys, 1961): 1-3 “Anecdotal”, 3-10 “Moderate”, 10-30 “Strong”, 30-100 “Very Strong”, > 100 "Extreme”.

To supplement BFs, Bayesian estimation analyses were conducted on focal effect sizes using the BEST package (Kruschke, 2014) in R. Ninety-five percent Highest Density Intervals (HDI), as well as the simulated mode effect size (i.e., the maximum $a$ posteriori estimate; MAP) are presented. Effect sizes associated with within-subject comparisons are Cohen's $d$ using $\mathrm{SD}_{\text {avg }}$ as the standardizer term (Cumming, 2012, p. 291) and generalized eta squared $\eta_{G}^{2}$ for more appropriate comparison to between-subject and within-subject effect sizes (see Bakeman, 2005). Frequentist statistics are also presented to facilitate interpretation of Bayesian analyses where appropriate.

\section{Performance}

Block Performance. Error trials were removed for RT analyses. In addition, outlier analysis was performed to exclude trial-level correct response times based on a 2.5 
standard deviation cut-off (Van Selst \& Jolicoeur, 1994). Using this criterion, 2.7\% of all trials were removed.

With respect to block RT, a 2 (Incentive) x 2 (Demand) mixed BF ANOVA was performed. First in reference to the error-only model, results demonstrated no evidence of a main effect of Incentive, $\mathrm{BF}_{\mathrm{NULL}}=3.3$, and extreme evidence for both a main effect of Demand and the interaction between Incentive and Demand $\left(\mathrm{BF}_{\mathrm{ALT}}>1,000\right.$ for both). We then compared the Demand main effect model to the Demand-by-Incentive interaction model. This comparison produced a BF of 19.91 in support of the Demand main effect model best fitting the block RT data relative to the interaction model. Correspondingly using NHST, the Demand main effect was significant, $F(1,197)=84.4$, $p<.001$, whereas the Incentive main effect, $F(1,197)=.71, p=.4, \eta_{G}^{2}=.003$, and the Demand-by-Incentive interaction were not, $F(1,197)=.002, p=.97, \eta_{G}^{2}<.0001$. Thus, RTs varied as a function of high- (70\% probability of switching) and low-demand (30\% probability of switching) with RTs being overall slower in the high-demand condition, and were not further modulated by incentive as expected.

With respect to block accuracy, a 2 (Incentive) x 2 (Demand) mixed BF ANOVA was performed. Merely anecdotal evidence was demonstrated in support of a main effect of Incentive, $\mathrm{BF}_{\mathrm{ALT}}=2.46$, a main effect of Demand, $\mathrm{BF}_{\mathrm{ALT}}=1.06$, and an interaction between Incentive and Demand, $\mathrm{BF}_{\mathrm{ALT}}=1.12$, relative to the error only model. Considering NHST, the main effect of Incentive did just reach significance, $F(1,197)=$ $5.4, p=.02, \eta_{G}^{2}=.02$, as did the main effect of Demand, $F(1,197)=4.76, p=.03, \eta_{G}^{2}=$ .003 . The interaction between Incentive and Demand did not reach significance, $F(1$, 
$197)=.76, p=.38, \eta_{G}^{2}=.0006$. Thus, there is little evidence (in terms of BFs) that accuracy varied as a function of Incentive or Demand. Though, NHST demonstrated a relatively small effect of incentive where accuracy was slightly better in the incentive condition. Furthermore, there was as small effect of demand in accuracy where accuracy was better in the low demand condition.

When considering overall performance in the first two forced-choice blocks, then, we find that RTs were slower in the high-demand condition where a $70 \%$ probability of a task-switch was faced relative to the low-demand option where a $30 \%$ probability of a task-switch was faced. Accuracy at the two tasks only slightly differed across the highand low-demand conditions and incentive conditions. In addition, RTs did not vary as a function of incentive. Next, we consider switch costs as an additional index of controlled processing.

Switch Costs. A 2 (Incentive) x 2 (Demand) x 2 (Switch/Repeat Trials) mixed BF ANOVA was performed on RTs and accuracy for switch trials. First considering evidence relative to the error model, there was extreme evidence (i.e., $\mathrm{BF}_{\mathrm{ALT}}>1,000$ ) for a main effect of Switch/Repeat Trials in RT, the two-way interaction of Switch/Repeat Trials and Demand, the two-way interaction between Incentive and Switch, and the threeway interaction between Incentive, Demand, and Switch/Repeat Trials. To determine the best fitting model to the data considering the four models above, BFs were sorted by magnitude and tested against one another. Overall, the interaction between Switch and Demand had the highest overall BF $\left(1.17 \times 10^{26}\right)$ and yielded BFs between 3.7 and 1854.6 against the competing higher-level models. Compared against the main effects of Switch and Demand, the interaction yielded a BF of $1.06 \times 10^{21}$ and $9.27 \times 10^{8}$ 
(respectively). Furthermore, NHST demonstrated a significant main effect of

Switch/Repeat Trials, $F(1,197)=95.75, p<.001, \eta_{G}^{2}=.05$, a two-way interaction between Switching and Demand, $F(1,197)=18.84, p<.001$, a non-significant two-way interaction between Incentive and Switch, $F(1,197)=.009, p=.92, \eta_{G}^{2}<.0001$, and a three-way interaction between Incentive, Demand, and Switch/Repeat Trials, $F(1,197)=$ $.15, p=.7, \eta_{G}^{2}<.0001$. Therefore, we can conclude with observed moderate to extreme evidence that the best fitting model of the RT data is the interaction between Switch and Demand confirming our prediction above. Specifically, switch costs were larger in the low-demand condition relative to the high-demand condition.

In terms of accuracy, there was evidence favoring the null hypothesis (i.e., the higher-level model did not differ from the random effect error model) for a main effect of Switch, $\mathrm{BF}_{\mathrm{NULL}}=6.25$, the two-way interaction between Incentive and Switch, $\mathrm{BF}_{\mathrm{NULL}}=$ 3.7, the two-way interaction between Switch and Demand, $\mathrm{BF}_{\mathrm{NULL}}>100$, and the threeway interaction between Incentive, Switch and Demand, $\mathrm{BF}_{\mathrm{NULL}}>1,000$. Thus, all BFs favored the null error model with at least positive evidence demonstrating that accuracy did not vary at any level as a function of switch/repeat trials. NHST confirmed this pattern, with all model terms being non-significant, all $F$ 's $<1.68$, all $p$ 's $>.2$.

When considering switching and demand in the first two forced-choice blocks, we find that switch costs in terms of RTs were smaller in the high-demand condition relative to the low-demand option, replicating the same pattern demonstrated elsewhere (Botvinick \& Rosen, 2009; Dunn \& Risko, submitted; Kool et al., 2010). We return to this pattern of RTs in the General Discussion. Again, accuracy did not vary as a function 
of demand and switch/repeat trials, and both RTs and accuracy did not vary as a function of incentive.

Table 1. Individuals' Low-Demand Selections and Descriptive Statistics for Performance in the Demand Selection Task

\begin{tabular}{rccccc}
\hline & \multicolumn{2}{c}{ Incentive } & & \multicolumn{2}{c}{ No Incentive } \\
\cline { 2 - 3 } \cline { 5 - 6 } \cline { 5 - 6 } & $\begin{array}{c}\text { Low Demand } \\
\text { Option }\end{array}$ & $\begin{array}{c}\text { High Demand } \\
\text { Option }\end{array}$ & & $\begin{array}{c}\text { Low Demand } \\
\text { Option }\end{array}$ & $\begin{array}{c}\text { High Demand } \\
\text { Option }\end{array}$ \\
\hline Block RT(ms) & $1195(261)$ & $1341(319)$ & & $1161(276)$ & $1299(314)$ \\
Repeat Trials & $1141(266)$ & $1287(332)$ & & $1109(280)$ & $1232(333)$ \\
Switch Trials & $1326(328)$ & $1369(344)$ & & $1284(336)$ & $1339(342)$ \\
Switch Costs & $185(253)$ & $82(244)$ & & $176(259)$ & $107(246)$ \\
Accuracy & $98.30 \%(12.93 \%)$ & $97.48 \%(15.67 \%)$ & & $96.59 \%(18.16 \%)$ & $96.24 \%(19.02 \%)$ \\
Repeat Trials & $98.44 \%(12.38 \%)$ & $97.46 \%(15.74 \%)$ & & $96.70 \%(17.88 \%)$ & $96.63 \%(18.05 \%)$ \\
Switch Trials & $97.98 \%(14.09 \%)$ & $97.49 \%(15.65 \%)$ & $96.35 \%(18.76 \%)$ & $96.07 \%(19.44 \%)$ \\
Switch Cost & $.46 \%(4.73 \%)$ & $.03 \%(5.09 \%)$ & & $.35 \%(5.78 \%)$ & $.56 \%(6.02 \%)$ \\
\hline
\end{tabular}

Note: The low-demand option consisted of a $30 \%$ probability of a task switch. The highdemand option consisted of a $70 \%$ probability of a task switch. Selections are presented for the low-demand option from the free-choice $\left(3^{\text {rd }}\right)$ block. Performance is presented for the forced-choice $\left(1^{\text {st }}\right.$ and $\left.2^{\text {nd }}\right)$ blocks. Standard deviations are in parentheses.

\section{Demand Selection Task}

First, to determine whether participants' less-effortful selections (i.e., selecting the $30 \%$ probability of a switch option) in the third free-choice block differed as a function of incentive, a Bayesian $t$-test as well as a Welch's independent samples $t$-test (Welch, 1947) was conducted. Results demonstrated only anecdotal evidence for the alternative hypothesis that the percentage of low-demand selections differed across the incentive conditions, $\mathrm{BF}_{\mathrm{ALT}}=.78, \mathrm{MAP} d=.27,95 \% \mathrm{HDI}[-.01, .55], t(195.18)=1.87, p=.06, d=$ 
$.26^{3}$. On average, those in the incentive condition selected the low-demand option $63 \%$ of the time $(S D=40 \%), \mathrm{BF}_{\mathrm{ALT}}=16.56, \mathrm{MAP} d=.35,95 \%$ HDI $[.14, .56], t(99)=$ $3.29, p=.001$. On average, individuals in the no incentive condition selected the lowdemand option $52 \%$ of the time, $\mathrm{BF}_{\mathrm{NULL}}=8.06$, MAP $d=.05,95 \%$ HDI $[-.15, .26]$, $t(100)=.48, p>.1,($ see Figure 2$)$.

Given known issues associated with averaging over individuals' choice patterns (e.g., Estes \& Maddox, 2005; Liew, Howe, \& Little, 2016) we additionally categorized participants into two groups: (1) demand-avoidant if they choose the low demand option more than $50 \%$ of the time, and (2) not demand-avoidance if they choose the low demand option less than (or equal to) $50 \%$ of the time. Sixty-eight percent $(n=68)$ of individuals were categorized as avoidant in the incentive group, whereas $52 \%(n=52)$ of individuals were categorized as avoidant in the no-incentive group. Results demonstrated anecdotal evidence that knowledge of an incentive influenced demand avoidance categorization $\mathrm{BF}_{\mathrm{ALT}}=2.82, \chi^{2}(1)=4.96, p=.03$. Specifically, extreme evidence was demonstrated that the percentage of demand avoidant individuals differed from chance in the incentive aware condition, $\mathrm{BF}_{\mathrm{ALT}}=120.64, \mathrm{MAP}=.67,95 \% \mathrm{HDI}[.58, .75], p<.001$ binomial test. Evidence for the null hypothesis was demonstrated for the incentive unaware condition, $\mathrm{BF}_{\mathrm{NULL}}=4.0, \mathrm{MAP}=.52,95 \%$ HDI $[.43, .62], p=.84$ binomial test (see Figure 2 ).

Overall the incentive condition did not differ in terms of avoidance relative to the no incentive condition (c.f., Figure 1), with the HDI suggesting only a small effect (i.e., that incentive increased avoidance) considered liberally. The same general pattern was demonstrated with respect to individuals being categorized as demand avoidant. Again, only anecdotal evidence was reached when considering avoiders and non-avoiders across 
the incentive conditions. Nonetheless, the rate of avoidance and the percentage of avoiders in the incentive condition was greater than chance.

Figure 2. Percentage of less-effortful selections and percentage of "Demand Avoiders" for incentive aware and unaware individuals.

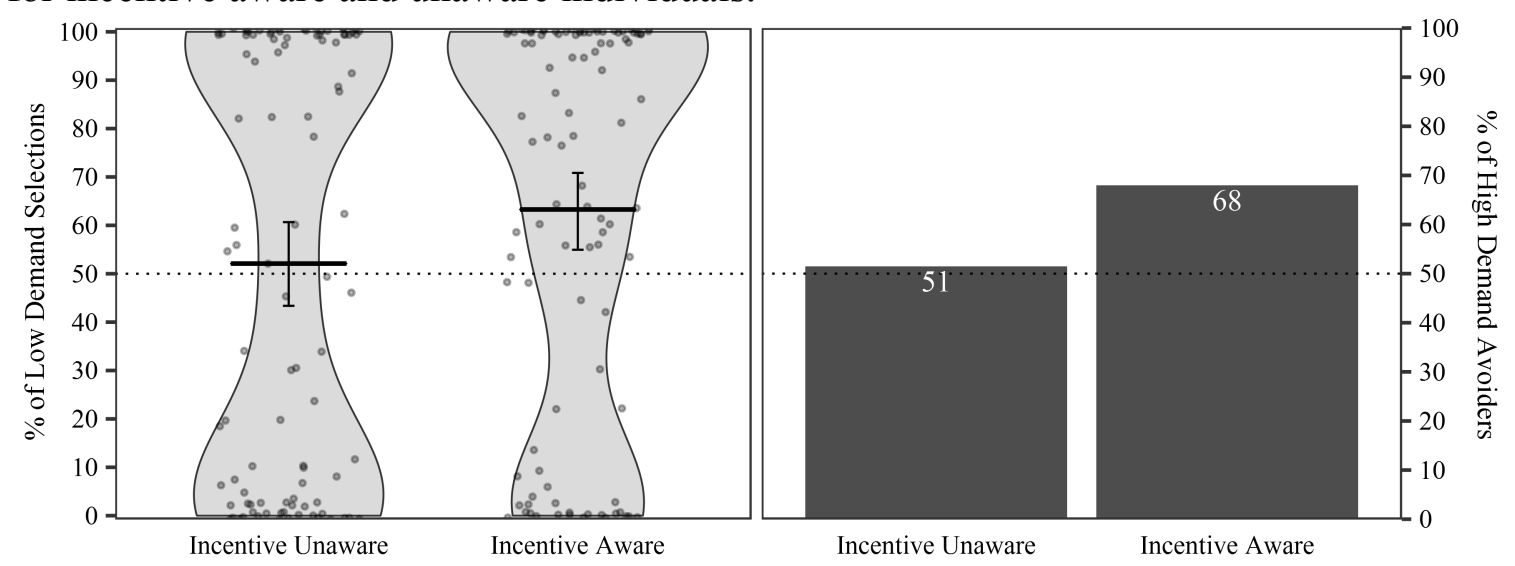

Note: the left panel displays a violin plot of the mean percentage (black band) of lowdemand selections (i.e., the $30 \%$ probability of a switch option) for the incentive condition and no incentive condition in the third free-choice block of the DST. Error bars represent $95 \%$ bias corrected and accelerated $(\mathrm{BCa})$ intervals. The right panel displays the percentage of individuals categorized as being a Demand Avoider for the incentive aware $(n=100)$ and unaware conditions $(n=99)$. The white number within the bars denotes the count of Demand Avoiders for each condition. The errors bar represents bias-corrected and accelerated bootstrapped 95\% confidence intervals of low-demand selections.

\section{No-loss Gambling Task}

To determine whether correct identification of the high switching option (i.e., a $70 \%$ probability of a switch) varied as a function of incentive, a chi-squared BF analysis was computed for the 2 (Incentive group) x 2 (Demand identification) count data. There was no evidence that identification of the high-switch deck varied across the incentive conditions, $\mathrm{BF}_{\mathrm{NULL}}=2.78, \chi^{2}(1)=1.22, p=.27$. Individuals in the incentive group correctly identified the high-switching deck $71 \%$ of the time, $\mathrm{BF}_{\mathrm{ALT}}>1,000, p<.001$ 
Binomial test, whereas the no-incentive group correctly identified the deck $62 \%$ of the time, $\mathrm{BF}_{\mathrm{ALT}}=4.8, p=.02$ Binomial test.

With respect to the decision to wager (i.e., taking the 50-50 gamble rather than sticking with one's answer to the switching question), a chi-squared BF analysis

demonstrated that wagering rates did not differ across the conditions, $\mathrm{BF}_{\mathrm{NULL}}=2.63, \chi^{2}$ $(1)=1.3, p=.25$. The incentive group gambled $39 \%$ of the time, $\mathrm{BF}_{\mathrm{ALT}}=2.4, p=.04$ Binomial test, and 30\% for the no-incentive group, $\mathrm{BF}_{\mathrm{ALT}}=395.7, p<.001$ Binomial test. Thus, most individuals stayed with their given answer to which option switched more in the DST across the incentive conditions.

We further examined the relation between the decision to wager and accuracy at identifying the high-switching deck, a 2 x 2 contingency table was analyzed crossing individuals' decision to gamble with their accuracy at identifying the high demand deck. The four cells generated from this table were: (1) Gamble | Incorrect, (2) Gamble | Correct, (3) No Gamble | Incorrect, and (4) No Gamble | Correct (see Table 2). There was no evidence demonstrated that accuracy in identifying the high switching option varied a function of deciding to gamble or not, $\mathrm{BF}_{\mathrm{NULL}}=1.82, \chi^{2}(1)=1.31, p=.25$. 
Table 2. Observed cell frequencies of individuals and sample sizes resulting from the crossing of gambling decision and identification of the high demand option.

\begin{tabular}{c|ccc}
\hline \multicolumn{2}{c}{} & \multicolumn{2}{c}{ Gambling Decision } \\
\cline { 3 - 3 } & & Gamble & Stay \\
Accurate at & Incorrect & $14 \%$ & $20 \%$ \\
$\begin{array}{c}\text { identifying } \\
\text { the high }\end{array}$ & & $n=27$ & $n=39$ \\
$\begin{array}{c}\text { demand } \\
\text { option? }\end{array}$ & Correct & $21 \%$ & $46 \%$ \\
& & $n=42$ & $n=91$ \\
\hline
\end{tabular}

\section{Awareness and Demand Avoidance}

Importantly, we next examined the association between correctly identifying the high-switching option, wagering, and avoiding demand. In the following, we ignore the incentive/no-incentive variable given the lack of effects reported above. Individuals lowdemand deck selections in the third free-choice block were analyzed using a one-factor (Gambling and Accuracy Categorization: Gamble | Incorrect, Gamble | Correct, No Gamble | Incorrect, and No Gamble | Correct) between subject ANOVA. This analysis yielded very strong evidence for a main effect of gambling and accuracy categorization, $\mathrm{BF}_{\mathrm{ALT}}=57.57, F(3,195)=5.83, p \leq .001, \eta_{G}^{2}=.08$. As apparent in Figure 3, individuals in the No Gamble | Correct category demonstrated significantly higher rates of demand avoidance relative to the three other categories, $\mathrm{BF}_{\mathrm{ALT}} \geq 6.60, p \leq .01$ for all comparisons. No other comparisons across the categories demonstrated positive evidence for the alternative, $\mathrm{BF}_{\mathrm{ALT}} \leq .35, p \geq .37$ for all comparisons.

Similar to above, we next assessed whether gambling and correctly identifying the high-switching option was independent of being categorized as demand avoidant via a $4 \mathrm{x}$ 2 contingency table. Participants demonstrated strong evidence that their accuracy and 
gambling decision were not independent of one another, $\mathrm{BF}_{\mathrm{ALT}}=28.53, \chi^{2}(3)=13.87, p$ $<.005$. Coinciding with the results for low-demand selections, there were more demand avoidant individuals in the No Gamble | Correct category relative to the other three categories, $\mathrm{BF}_{\mathrm{ALT}} \geq 4.83, p \leq .01$ for all comparisons. No other comparisons across the categories demonstrated positive evidence for the alternative, $\mathrm{BF}_{\mathrm{ALT}} \leq .44, p \geq .69$ for all comparisons (see Figure 3).

Individuals that correctly identified the high-switching option and did not gamble on their judgment (i.e., the No Gamble | Correct) category highly yielded higher rates of demand avoidance. This category can be considered (relatively) the "most" aware of the four categories. The other three categories yielded rates of demand avoidance at chance whether indexed by percentage of low-demand selections or percentage of demand avoiders. Specifically, individuals that did not correctly identify the high switching deck though were confident in their choice (i.e., the No Gamble | Incorrect category) overall demonstrated avoidance near chance. Similarly, both categories that can be considered to have guessed on the state of their experience in the DST (i.e., Gamble | Incorrect, Gamble | Correct) overall demonstrated avoidance near chance. Thus, becoming aware of the correct difference between the options in terms of switching and having high confidence in that experience appeared to work conjunctively in driving effort avoidance above chance levels. 
Figure 3. Percentage of less-effortful selections and percentage of "Demand Avoiders" for gambling behavior and high-demand identification accuracy categorization.

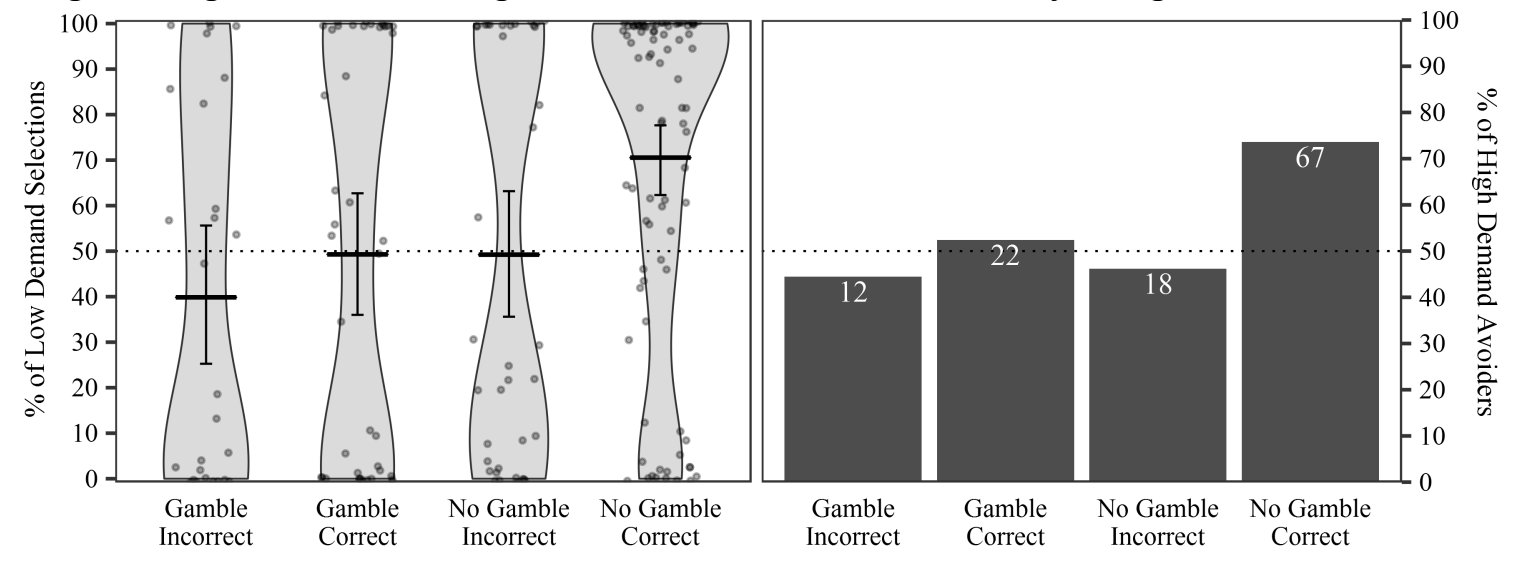

Note: the left panel displays a violin plot of the mean percentage (black band) of lowdemand selections (i.e., the $30 \%$ probability of a switch option) for the four cells based on the crossing of individuals gambling decision and accuracy at identifying the highdemand option. Error bars represent $95 \%$ bias corrected and accelerated (BCa) intervals. The right panel displays the percentage of individuals categorized as being a Demand Avoider for these four conditions. The white number within the bars denotes the count of Demand Avoiders for each condition (see Table 2 for cell $n$ 's). The errors bar represents bias-corrected and accelerated bootstrapped 95\% confidence intervals of low-demand selections.

\section{Non-preregistered Analyses}

A straightforward prediction following from the above results is that individuals that become aware of differences in demand produce different levels of performance when engaging the different options. As an example, individuals that become aware may show differential effects in response to increases in demands in terms of control such as larger preparation effects to handle frequent task-switching (Monsell, 2003). This modulation of control may then lead to an increase in metacognitive awareness of the demand difference across options. To examine this, we conducted additional analyses on performance using gambling and accuracy categorization as a factor. We note here that all of the following analyses were not preregistered. 
A 4 (Gambling and Accuracy Categorization) x 2 (Demand) mixed-design ANOVA was used to assess whether individuals' demand effect (i.e., the difference between the high- and low-demand options) varied as a function of level of awareness. A direct comparison of the interaction model against the error model produced extreme evidence for the alternative, $\mathrm{BF}_{\mathrm{ALT}}>1,000$. Furthermore, a comparison of the interaction model against the simple main effect model of Demand demonstrated extreme evidence that the main effect model better described the data, $\mathrm{BF}_{\mathrm{ALT}}=137.5$. This demonstrates that there were not differential demand effects across the four categories. NHST

additionally confirmed a non-significant interaction, $F(3,195)=1.62, p=.19, \eta_{G}^{2}=.004$.

Furthermore, performing the same analysis adding Switch/Repeat Trials as a factor demonstrated that the interaction model, though showing extreme evidence for the alternative relative to the error-only mode, $\mathrm{BF}_{\mathrm{ALT}}>1,000$, was outperformed by 11 of the other models (out of 17). The model of a Demand and Switch/Repeat main effect demonstrated extreme evidence in better describing the data, $\mathrm{BF}_{\mathrm{ALT}}=1.3 \times 10^{9}$. Furthermore, this dual main effect model demonstrated strong evidence in outperforming the three main effect model (inclusion of gambling and accuracy categorization), $\mathrm{BF}_{\mathrm{ALT}}=$ 19.88. Again, NHST confirmed this with a non-significant three-way interaction $F(3$, $195)=.86, p=.46, \eta_{G}^{2}=.0007$. Thus, following the above demand effect analysis, there were not differential switch costs across the four categorizations (see Table 3). 
Table 3. Mean response times for gambling behavior and high-demand identification accuracy categorization.

\begin{tabular}{ccccc}
\hline & Gamble | Incorrect & Gamble $\mid$ Correct & No Gamble | Incorrect & No Gamble | Correct \\
\cline { 2 - 5 } Low-Demand & & & & \\
Block RT (ms) & $1459(1258)$ & $1252(1020)$ & $1260(952)$ & $1246(990)$ \\
Repeat Trials & $1404(1991)$ & $1170(943)$ & $1189(889)$ & $1179(947)$ \\
Switch Trials & $1567(1376)$ & $1414(1143)$ & $1416(1060)$ & $1406(1072)$ \\
Switch Costs & $163(281)$ & $244(212)$ & $227(231)$ & $227(272)$ \\
High-Demand & & & & \\
Block RT (ms) & $1648(1648)$ & $1391(1148)$ & $1355(980)$ & $1480(1206)$ \\
Repeat Trials & $1561(1491)$ & $1304(917)$ & $1294(950)$ & $1369(1148)$ \\
Switch Trials & $1689(1690)$ & $1431(1238)$ & $1382(992)$ & $1533(1229)$ \\
Switch Costs & $128(318)$ & $127(179)$ & $88(207)$ & $164(260)$ \\
\hline
\end{tabular}

Note: standard deviations are presented in parentheses.

\section{General Discussion}

The present investigation sought to extend the hypothesis, under a cue-based metacognitive framework of cognitive effort, that awareness of effort cues is a prerequisite to avoiding effortful lines of action. In a preregistered study, we attempted to moderate levels of avoidance by introducing an incentive that was focused on having individuals monitor the task. First, we did not find differences in performance across the incentive conditions. Though individuals in the incentive condition were categorized as being demand avoidant above chance-level, we did not observe differences statistically across the incentive conditions in either rates of demand avoidance or percentage of individuals categorized as avoiders. Nonetheless, when considering awareness through the no-loss gambling task, it was fairly clear that individuals that correctly identified the high-switching option and did not gamble (i.e., the relatively highest level of awareness of the switching cue), demonstrated demand avoidance above chance. In the following 
we consider the current results within a cue-based metacognitive account of cognitive effort and consider future directions for the study of cognitive effort.

Cue-Based Metacognition of Cognitive Effort

The cue-based metacognitive account of cognitive effort posits that individuals monitor variation across lines of action by way of cues, and utilize these cues to infer demand when making effort-based decisions (i.e., avoiding effort). One distinct prediction made from this account is that, given cognitive effort is the result of a metalevel evaluation, individuals may need to be consciously aware of cues to make these types of decisions. That is, effort cues must reach a particular level of conscious awareness (e.g., Block, 1995) in order to factor into an evaluation of effort. Some initial evidence supports this hypothesis (Desender et al., 2017), and the current experiment overall adds additional credence to the claim.

Our specific attempted manipulation at moderating levels of avoidance was relatively unsuccessful at pushing rates of avoidance above those in the no incentive condition. Nonetheless, individuals in the incentive condition overall demonstrated avoidance above chance, whereas this was not the case for those in the no incentive condition. The resulting null effect (or small effect at best), was likely due to the incentive manipulation being purposely broad to attempt to leave performance unaffected across conditions. We were successful at keeping performance similar across the conditions. Though, the manipulation may have been too general for individuals, and attention may have been paid to different aspects of the task other than the frequency of switching (e.g., the colors of the response items). We would expect, however, that stronger manipulations, perhaps explicitly signaling differences between the choice 
options (e.g., Gold et al., 2014), would exacerbate levels of awareness, and thus, increase effort avoidance.

Outside of the incentive manipulation we did find clear evidence that individuals who noticed a difference in switching across the options and were confident in that experience (i.e., $46 \%$ of the current sample in the No Gamble | Correct category) overall demonstrated rates of demand avoidance above chance. This was not the case for the other three categories, suggesting that awareness indeed plays an important role in effort avoidance. Moreover, indices of control (i.e., performance) were similar across all of the categories. The interaction between switch/repeat trials and low/high demand (i.e., switch costs were smaller in the high-demand option relative to the low-demand option) signifies that control processes related to task-switching were deployed. Within the context of a dual mechanism framework of control (Braver, 2012), smaller switch costs in the highswitching context can be considered to indicate the use of a more proactive mode of control (e.g., maintaining both tasks-sets in a partially activated state) when switching occurs frequently. Importantly, this occurred irregardless of awareness of the difference in switching across the options (i.e., a $70 \%$ probability versus a $30 \%$ probability). Therefore, differential control for nearly half of the current sample proceeded without awareness of the difference in the specific control-related cue. This coincides, with work outlined in the introduction that control can be deployed without awareness. Awareness, however, appears to be required for the avoidance of the demand related to the engagement of that control.

Following from the current results, key questions then to consider is how individuals become aware of cues when situated in a task (e.g., Dehaene \& Changeux, 
2011) and utilize cues when making effort-based decisions. Though differing in specification, extant accounts of conscious access posit that awareness proceeds in a serial manner and is the result of an assembly of information that can be shared across a "workspace" (for a review see Dehaene \& Changeux, 2011). For example, the dynamic core hypothesis (Tononi \& Edelman, 1998) states that information only becomes accessible once encoded information achieves differentiation (i.e., the isolation of specific content out of much other potential content) and integration (i.e., the formation of a coherent representation). Within the Global Neuronal Workspace (GNW) model (Dehaene, Sergent, \& Changeux, 2003; Dehaene \& Naccache, 2001), stimulus-relevant information supported by feedback and feed-forward connection is "ignited" into a global state of consciousness. At these points of conscious awareness, representations (e.g., cues) can then be selected for further metacognitive processes (Snodgrass et al., 2004) largely supported by various sub-regions of the PFC (e.g., the rostrolateral PFC, Fleck et al., 2006; Medalla \& Barbas, 2010; ventral medial PFC, Hogan, Galaro, \& Chib, 2017; Schnyer, Nicholls, \& Verfaellie, 2005; lateral PFC, Mcguire \& Botvinick, 2010; left dorsolateral PFC, Lau \& Passongham, 2006).

Thus, conscious access seems to be associated with the need to accumulate evidence and some form of criterion where information produces reasonable confidence (or reliability; Michaelian, 2009; 2012; Yeung \& Summerfield, 2012) and is deemed task-relevant (Snodgrass et al., 2004). Applied to the present context, individuals in the No Gamble | Correct category may have accumulated evidence related to the frequency of task switching in each deck and more importantly the difference across decks at different rates. Indeed, there is evidence of individual differences in detecting differences 
in effort cues (i.e., more/less switching) across lines of action related to IQ (Gold et al., 2014). Or individual differences may exist in decision criterion setting for determining whether a cue is reliable enough to signal a difference in effort (e.g., individuals' $\beta$ values in Signal Detection Theory, Long \& Shelnutt, 1973). Thus, both processes may serve as important directions for future research in effort-based decision-making, especially when considering cue awareness as a determinant of action selection. Aggregate measures of awareness would be especially useful, including behavioral, physiological, and anatomical indices (Seth, Dienes, Cleeremans, Overgaard, \& Pessoa, 2008), to determine what leads individuals to become aware of cues, and utilizing those cues, in effort avoidance.

Cue-based Inference of Effort versus Sheer Subjective Feelings of Effort

There is the important case of the subsets of individuals that demonstrated demand avoidance in the three categories that can be considered to be less aware than the No Gamble I Correct category (c.f., Figure 3). Straightforwardly, it may be that given these individuals were putatively unaware to some degree of the switching cue that they simply chose an option at random in the third free-choice block, leading to choices occurring around chance for the three categories. Alternatively, it may possible that, although overall choices were at chance, individuals became partially aware of some aspect of demand, though this information was unreportable (or was reported incorrectly) in the gambling task. That is, some information may have been experienced at the phenomenal level, but did not meet the conditions (e.g., high evidence, see above) to move to the higher-level access consciousness where evaluations could then be made (Block, 1995). 
As noted in the introduction, research on metacognition emphasizes the difference between contributions of processes that operate within full consciousness (i.e., cueutilization) and below full consciousness (i.e., a sheer subjective feeling; Kelley \& Jacoby, 1996; Koriat, 2000; Koriat, Nussinson, Bless, \& Shaked, 2008). Reliance on a sheer subjective feeling of effort may have been the case for individuals that did not correctly identify the high switching deck though were confident in their choice (i.e., the No Gamble | Incorrect category). For example, individuals in this category demonstrated avoidance at chance. This group may have felt a difference in effort across the options during the DST, but lacked the level of awareness to determine the correct source of the difference (e.g., the difference in switching; Dunn et al., 2016) and randomly inferred one alternative was more effortful in the third block. Furthermore, the potential reliance on a sheer subjecting of feeling of effort then led to high confidence in their choice (i.e., choosing not to gamble). That is, for these individuals they felt a difference in effort which they were confident about, though they were unable to directly map the difference. As noted above, aggregate measures of awareness would help shed light on the processes associated with partial awareness of aspects of tasks and effort avoidance.

\section{Conclusion}

Cognitive effort has benefited from a recent resurgence in inter ests from researchers. One important goal of these endeavors is to understand how individuals make effort-based decisions. The present study looked to further understand this decision processes. Overall, awareness does appear to be an important component of evaluating cognitive effort. This proposal opens several doors for future research focused on the relation between effort and consciousness. 


\section{References}

Aarts, E., Roelofs, A., Franke, B., Rijpkema, M., Fernández, G., Helmich, R. C., \& Cools, R. (2010). Striatal dopamine mediates the interface between motivational and cognitive control in humans: evidence from genetic imaging. Neuropsychopharmacology, 35(9), 1943-1951.

Abrahamse, E., \& Braem, S. (2015). Experience a conflict-either consciously or not (commentary on Desender, Van Opstal, and Van den Bussche, 2014). Frontiers in Psychology, 6, 179.

Bakeman, R. (2005). Recommended effect size statistics for repeated measures design. Behavior Research Methods, 37(3), 379-384.

Block, N. (1995). On a confusion about a function of consciousness. Behavioral and Brain Sciences, 18(2), 227-247.

Botvinick, M. M. (2007). Conflict monitoring and decision making: Reconciling two perspectives on anterior cingulate function. Cognitive, Affective, \& Behavioral Neuroscience, 7(4), 356-366.

Botvinick, M. M, Braver, T. S. (2015). Motivation and cognitive control: From behavior to neural mechanism. Annual Review of Psychology, 66, 83-113.

Botvinick, M. M., Huffstetler, S., \& McGuire, J. T. (2009). Effort discounting in human nucleus accumbens. Cognitive, Affective, \& Behavioral Neuroscience, 9(1), 16-27

Botvinick, M. M., \& Rosen, Z. B. (2009). Anticipation of cognitive demand during decision-making. Psychological Research PRPF, 73(6), 835-842. 
Braver, T. S. (2012). The variable nature of cognitive control: a dual mechanisms framework. Trends in Cognitive Sciences, 16(2), 106-113.

Bugg, J. M., \& Diede, N. T. (2017). The effects of awareness and secondary task demands on Stroop performance in the pre-cued lists paradigm. Acta Psychologica. Advanced online publication. DOI:

doi.org/10.1016/j.actpsy.2016.12.013

Brainard, D. H., \& Vision, S. (1997). The psychophysics toolbox. Spatial Vision, 10, 433436.

Cleeremans, A., Timmermans, B., \& Pasquali, A. (2007). Consciousness and metarepresentation: a computational sketch. Neural Networks, 20(9), 1032-1039.

Cochran, W. (1952). The $\chi 2$ Test of Goodness of Fit. The Annals of Mathematical Statistics, 23(3), 315-345.

Cohen, M. X., Simon van Gaal, K., \& Lamme, V. A. (2009). Unconscious errors enhance prefrontal-occipital oscillatory synchrony. Frontiers in Human Neuroscience, 3, 54.

Cumming, G. (2012). Understanding the new statistics: Effect sizes, confidence intervals, and meta-analysis. New York, NY: Routledge.

Crump, M. J., Gong, Z., \& Milliken, B. (2006). The context-specific proportion congruent Stroop effect: Location as a contextual cue. Psychonomic Bulletin \& Review, 13(2), 316-321.

Dehaene, S., \& Changeux, J. P. (2011). Experimental and theoretical approaches to conscious processing. Neuron, 70(2), 200-227. 
Dehaene, S., \& Naccache, L. (2001). Towards a cognitive neuroscience of consciousness: basic evidence and a workspace framework. Cognition, 79(1), 1-37.

Dehaene, S., Sergent, C., \& Changeux, J. P. (2003). A neuronal network model linking subjective reports and objective physiological data during conscious perception. Proceedings of the National Academy of Sciences, 100(14), 8520 8525

Del Cul, A., Baillet, S., \& Dehaene, S. (2007). Brain dynamics underlying the nonlinear threshold for access to consciousness. PLoS Biology, 5(10), e260.

de Ridder, D. T., Lensvelt-Mulders, G., Finkenauer, C., Stok, F. M., \& Baumeister, R. F. (2012). Taking stock of self-control: A meta-analysis of how trait self-control relates to a wide range of behaviors. Personality and Social Psychology Review, 16(1), 76-99.

Desender, K., Buc Calderon, C., Van Opstal, F., \& Van den Bussche, E. (2017). Avoiding the conflict: Metacognitive awareness drives the selection of low demand contexts. Journal of Experimental Psychology: Human Perception and Performance, 43(7), 1397-1410.

Desender K., van den Bussche E. (2012). Is consciousness necessary for conflict adaptation? A state of the art. Frontiers in Human Neuroscience, 6, 3.

Desender, K., Van Lierde, E., \& Van den Bussche, E. (2013). Comparing conscious and unconscious conflict adaptation. PLoS One, 8(2), e55976.

Desender, K., Van Opstal, F., \& van den Bussche, E. (2014). Feeling the conflict: The crucial role of conflict experience in adaptation. Psychological Science, 25(3), $675-683$. 
Diede, N. T., \& Bugg, J. M. (2017). Cognitive effort is modulated outside of the explicit awareness of conflict frequency: evidence from pupillometry. Journal of Experimental Psychology: Learning, Memory, and Cognition, 43(5), 824-835.

Dienes, Z., \& Altmann, G. (1997). Transfer of implicit knowledge across domains: How implicit and how abstract? In D. Berry (Ed.), How implicit is implicit learning (pp. 107-123). New York: Oxford University Press.

Dienes, Z., Altmann, G., Kwan, L., \& Goode, A. (1995). Unconscious knowledge of artificial grammars is applied strategically. Journal of Experimental Psychology: Learning, Memory, and Cognition, 21(5), 1322-1338.

Dienes, Z., Broadbent, D., \& Berry, D. C. (1991). Implicit and explicit knowledge bases in artificial grammar learning. Journal of Experimental Psychology: Learning, Memory, and Cognition, 17(5), 875-887.

Dienes, Z., \& Perner, J. (1999). A theory of implicit and explicit knowledge. Behavioral and Brain Sciences, 22(5), 735-808.

Dienes, Z., \& Seth, A. (2010). Gambling on the unconscious: A comparison of wagering and confidence ratings as measures of awareness in an artificial grammar task. Consciousness and Cognition, 19(2), 674-681.

Dunn, T. L., Lutes, D. J. C., \& Risko, E. F. (2016). Metacognitive evaluation in the avoidance of demand. Journal of Experimental Psychology: Human Perception and Performance, 42(9), 1372-1387.

Dunn, T. L., \& Risko, E. F. (2015). Toward a metacognitive account of cognitive offloading. Cognitive Science, 40(5), 1080-1127. 
Dunn, T. L., \& Risko, E. F. (submitted). Understanding the Cognitive Miser: Cue utilization in Effort Avoidance. https://www.researchgate.net/publication/303543690_Understanding_the_Cogni ve_Miser_Cue-utilization_in_Effort_Avoidance

Eriksen, B. A., \& Eriksen, C. W. (1974). Effects of noise letters upon the identification of a target letter in a nonsearch task. Attention, Perception, \& Psychophysics, 16(1), 143-149.

Estes, W. K., \& Maddox, W. T. (2005). Risks of drawing inferences about cognitive processes from model fits to individual versus average performance. Psychonomic Bulletin \& Review, 12(3), 403-408.

Fleck, M. S., Daselaar, S. M., Dobbins, I. G., \& Cabeza, R. (2006). Role of prefrontal and anterior cingulate regions in decision-making processes shared by memory and nonmemory tasks. Cerebral Cortex, 16, 1623-1630.

Fleming, S. M., \& Dolan, R. J. (2012). The neural basis of metacognitive ability. Philosophical Transactions of the Royal Society B, 367(1594), 1338 1349.

Gold, J. M., Kool, W., Botvinick, M. M., Hubzin, L., August, S., \& Waltz, J. A. (2014). Cognitive effort avoidance and detection in people with schizophrenia. Cognitive, Affective, \& Behavioral Neuroscience, 15(1), 145-154.

Gómez, R. L., \& Gerken, L. (2000). Infant artificial language learning and language acquisition. Trends in Cognitive Sciences, 4(5), 178-186. 
Gratton, G., Coles, M. G., \& Donchin, E. (1992). Optimizing the use of information: strategic control of activation of responses. Journal of Experimental Psychology: General, 121(4), 480-506.

Hagger, M. S. (2013). The opportunity cost model: Automaticity, individual differences, and self-control resources. Brain and Behavioral Sciences, 36(6), 687-688.

Heinemann, A., Kunde, W., \& Kiesel, A. (2009). Context-specific prime-congruency effects: On the role of conscious stimulus representations for cognitive control. Consciousness and Cognition, 18(4), 966-976.

Hester, R., Foxe, J. J., Molholm, S., Shpaner, M., \& Garavan, H. (2005). Neural mechanisms involved in error processing: a comparison of errors made with and without awareness. Neuroimage, 27(3), 602-608.

Hogan, P. S., Galaro, J. K., \& Chib, V. S. (2017). Dissociable roles of ventromedial prefrontal cortex and anterior cingulate in subjective valuation of prospective effort. bioRxiv, 079467.

Inzlicht, M., Schmeichel, B. J., \& Macrae, C. N. (2014). Why self-control seems (but may not be) limited. Trends in Cognitive Sciences, 18(3), 127-133.

Jeffreys, H. (1961). Theory of probability (3rd ed.). Oxford: Oxford University Press, Clarendon Press.

Joshi, S., Li, Y., Kalwani, R. M., \& Gold, J. I. (2016). Relationships between pupil diameter and neuronal activity in the locus coeruleus, colliculi, and cingulate cortex. Neuron, 89(1), 221-234.

Kelley, C. M., \& Jacoby, L. L. (1996). Adult egocentrism: Subjective experience versus analytic bases for judgment. Journal of Memory and Language, 35(2), 157-175. 
Klein, T. A., Endrass, T., Kathmann, N., Neumann, J., von Cramon, D. Y., \& Ullsperger, M. (2007). Neural correlates of error awareness. Neuroimage, 34(4), 1774-1781.

Kleiner, M., Brainard, D., Pelli, D., Ingling, A., Murray, R., \& Broussard, C. (2007). What's new in Psychtoolbox-3. Perception, 36, ECVP Abstract Supplement.

Kool, W., \& Botvinick, M. M. (2014). A labor/leisure tradeoff in cognitive control. Journal of Experimental Psychology: General, 143(1), 131-141.

Kool, W., McGuire, J. T., Rosen, Z. B., \& Botvinick, M. M. (2010). Decision making and the avoidance of cognitive demand. Journal of Experimental Psychology: General, 139(4), 665-682.

Kool, W., McGuire, J. T., Wang, G. J., \& Botvinick, M. M. (2013). Neural and behavioral evidence for an intrinsic cost of self-control. PloS One, 8(8), e72626.

Koriat, A. (1997). Monitoring one's own knowledge during study: A cue-utilization approach to judgments of learning. Journal of Experimental Psychology: General, 126(4), 349-370.

Koriat, A. (2000). The feeling of knowing: Some metatheoretical implications for consciousness and control. Consciousness and Cognition, 9(2), 149-171.

Koriat, A., Nussinson, R., Bless, H., \& Shaked, N. (2008). Information-based and experience-based metacognitive judgments: Evidence from subjective confidence. In J. Dunlosky \& R. A. Bjork (Eds.), Handbook of memory and metamemory (pp. 117-135). New York, NY: Psychology Press.

Kruschke, J. K. (2013). Bayesian estimation supersedes the t test. Journal of Experimental Psychology: General, 142(2), 573-603. 
Kurzban, R., Duckworth, A., Kable, J. W., \& Myers, J. (2013). An opportunity cost model subjective effort and task performance. Behavioral and Brain Sciences, 36, $661-726$.

Lau, H. C. (2008). Are we studying consciousness yet? In L. Weiskrantz \& M. David (Eds.), Frontiers of consciousness: Cichele lectures (pp. 245-258). Oxford: Oxford University Press.

Lau, H. C., \& Passingham, R. E. (2007). Unconscious activation of the cognitive control system in the human prefrontal cortex. Journal of Neuroscience, 27(21), 5805 5811.

Lee, M. D., \& Wagenmakers, E. J. (2013). Bayesian data analysis for cognitive science: A practical course. New York, NY: Cambridge University Press.

Liew, S. X., Howe, P. D., \& Little, D. R. (2016). The appropriacy of averaging in the study of context effects. Psychonomic Bulletin \& Review, 23(5), 1639-1646.

MacLeod, C. M. (1991). Half a century of research on the Stroop effect: an integrative review. Psychological Bulletin, 109(2), 163-203.

McGuire, J. T., \& Botvinick, M. M. (2010). Prefrontal cortex, cognitive control, and the registration of decision costs. Proceeding of the National Academy of Sciences of the United States of America, 107, 7922-7926.

Medalla, M., \& Barbas, H. (2010). Anterior cingulate synapses in prefrontal areas 10 and 46 suggest differential influence in cognitive control. Journal of Neuroscience, 30(48), 16068-16081.

Michaelian, K. (2009). Reliabilism and privileged access. Journal of Philosophical Research, 34, 69-109. 
Michaelian, K. (2012). Metacognition and endorsement. Mind \& Language, 27(3), 284 307.

Monsell, S. (2003). Task switching. Trends in Cognitive Sciences, 7(3), 134-140.

Monsell, S., Yeung, N., \& Azuma, R. (2000). Reconfiguration of task-set: Is it easier to switch to the weaker task? Psychological research, 63(3), 250-264.

Morey, R. D., \& Rouder, J. N. (2015). BayesFactor: Computation of Bayes Factors for Common Designs. R package version 0.9.11-1. Retrieved from http:/CRAN.Rproject.org/package $=$ BayesFactor

Mueller, M. L., Dunlosky, J., \& Tauber, S. K. (2016). The effect of identical word pairs on people's metamemory judgments: What are the contributions of processing fluency and beliefs about memory?. The Quarterly Journal of Experimental Psychology, 69(4), 781-799.

Mulert, C., Menzinger, E., Leicht, G., Pogarell, O., \& Hegerl, U. (2005). Evidence for a close relationship between conscious effort and anterior cingulate cortex activity. International Journal of Psychophysiology, 56(1), 65-80.

Naccache, L., Dehaene, S., Cohen, L., Habert, M. O., Guichart-Gomez, E., Galanaud, D., \& Willer, J. C. (2005). Effortless control: executive attention and conscious feeling of mental effort are dissociable. Neuropsychologia, 43(9), 1318-1328.

Nelson T. O., Narens, L. (1990). Metamemory: a theoretical framework and new findings. In G.H. Bower (Ed.), The psychology of learning and motivation: advances in research and theory (Vol 26, pp. 125-173). New York, NY: Academic. 
Overgaard, M., \& Sandberg, K. (2012). Kinds of access: different methods for report reveal different kinds of metacognitive access. Philosophical Transactions of the Royal Society B: Biological Sciences, 367(1594), 1287-1296.

Payne, J. W., Bettman, J. R., \& Johnson, E. J. (1993). The adaptive decision maker. New York City, NY: Cambridge University Press.

Pelli, D. G. (1997). The VideoToolbox software for visual psychophysics: Transforming numbers into movies. Spatial Vision, 10(4), 437-442.

Pennycook, G., Fugelsang, J. A., \& Koehler, D. J. (2015). What makes us think? A three stage dual-process model of analytic engagement. Cognitive Psychology, 80, 34 72.

Questienne, L., Van Opstal, F., van Dijck, J. P., \& Gevers, W. (2016). Metacognition and cognitive control: Behavioural adaptation requires conflict experience. The Quarterly Journal of Experimental Psychology, 1-15.

R Core Team (2014). R: A language and environment for statistical computing. R Foundation for Statistical Computing, Vienna, Austria. Retrieved from http://www.R-project.org

Ratcliff, R. (1978). A theory of memory retrieval. Psychological Review, 85(2), 59-108.

Ratcliff, R., \& McKoon, G. (2008). The diffusion decision model: theory and data for two-choice decision tasks. Neural Computation, 20(4), 873-922.

Reber, A. S. (1967). Implicit learning of artificial grammars. Journal of Verbal Learning and Verbal Behavior, 6(6), 855-863.

Reber, A. S. (1976). Implicit learning of synthetic language. Journal of Experimental Psychology: Human Learning and Memory, 2, 88-94. 
Reber, A. S. (1989). Implicit learning and tacit knowledge. Journal of Experimental Psychology: General, 118, 219-235.

Reber, A. S., Allen, R., \& Regan, S. (1985). Syntactical learning and judgment, still unconscious and still abstract: comment on Dulany, Carlson, and Dewey. Journal of Experimental Psychology: General, 114(1), 17-24.

Reber, A. S., \& Lewis, S. (1977). Implicit learning: An analysis of the form and structure of a body of tacit knowledge. Cognition, 5(4), 333-361.

Reder, L. M. (1988). Strategic control of retrieval strategies. In G. Bower (Ed.), The psychology of learning and motivation (Vol. 22, pp. 227-259). New York, NY: Academic Press.

Reder, L. M., \& Schuun, C. D. (1996). Metacognition does not imply awareness: Strategy choice is governed by implicit learning and memory. In L. M. Reder (Ed.), Implicit memory and metacognition (pp. 45-78). Hillsdale, NJ: Erlbaum.

Reuss, H., Desender, K., Kiesel, A., \& Kunde, W. (2014). Unconscious conflicts in unconscious contexts: the role of awareness and timing in flexible conflict adaptation. Journal of Experimental Psychology: General, 143(4), 1701-1718.

Risko, E. F., \& Gilbert, S. J. (2016). Cognitive offloading. Trends in Cognitive Sciences, 20(9), 676-688.

Risko, E. F., \& Stolz, J. A. (2010). The proportion valid effect in covert orienting: Strategic control or implicit learning? Consciousness and cognition, 19(1), 432 442.

Rouder, J. N. (2014). Optional stopping: No problem for Bayesians. Psychonomic Bulletin \& Review, 21(2), 301-308. 
Rouder, J. N., Morey, R. D., Speckman, P. L., \& Province, J. M. (2012). Default Bayes factors for ANOVA designs. Journal of Mathematical Psychology, 56(5), 356 374.

Schmidt, J. R., Crump, M. J., Cheesman, J., \& Besner, D. (2007). Contingency learning without awareness: Evidence for implicit control. Consciousness and Cognition, 16(2), 421-435.

Schönbrodt, F. D., Wagenmakers, E. J., Zehetleitner, M., \& Perugini, M. (2017). Sequential hypothesis testing with Bayes factors: Efficiently testing mean differences. Psychological Methods, 22(2), 322-339.

Schouppe, N., de Ferrerre, E., Van Opstal, F., Braem, S., \& Notebaert, W. (2014). Conscious and unconscious context-specific cognitive control. Frontiers in Psychology, 5, 539.

Schurger, A., \& Sher, S. (2008). Awareness, loss aversion, and post-decision wagering. Trends in Cognitive Sciences, 12(6), 209-210.

Schnyer, D. M., Nicholls, L., \& Verfaellie, M. (2005). The role of VMPC in metamemorial judgments of content retrievability. Journal of Cognitive Neuroscience, 17(5), 832-846.

Seth, A. K., Dienes, Z., Cleeremans, A., Overgaard, M., \& Pessoa, L. (2008). Measuring consciousness: relating behavioural and neurophysiological approaches. Trends in Cognitive Sciences, 12(8), 314-321.

Shenhav, A., Botvinick, M. M., \& Cohen, J. D. (2013). The expected value of control: an integrative theory of anterior cingulate cortex function. Neuron, 79(2), 217-240. 
Shenhav, A., Musslick, S., Lieder, F., Kool, W., Griffiths, T. L., Cohen, J. D., \& Botvinick, M. M. (2017). Toward a rational and mechanistic account of mental effort. Annual Review of Neuroscience, 40, 99-124.

Shimamura, A. P. (2000). Toward a cognitive neuroscience of metacognition. Consciousness and Cognition, 9(2), 313-323.

Shimamura, A. P. (2008). A neurocognitive approach to metacognitive monitoring and control. In J. Dunlosky \& R. A. Bjork (Eds.), Handbook of metamemory and memory (pp. 373-390). New York: Psychology Press.

Simmons, J. P., Nelson, L. D., \& Simonsohn, U. (2012). A 21 Word Solution. Dialogue, The Official Newsletter of the Society for Personality and Social Psychology, 26(2), 4-7.

Skosnik, P. D., Mirza, F., Gitelman, D. R., Parrish, T. B., Mesulam, M. M., \& Reber, P. J. (2002). Neural correlates of artificial grammar learning. Neuroimage, 17(3), $1306-1314$.

Snodgrass, M., Bernat, E., \& Shevrin, H. (2004). Unconscious perception: A model based approach to method and evidence. Attention, Perception, \& Psychophysics, 66(5), 846-867.

Snodgrass, M., Kalaida, N., \& Winer, E. S. (2009). Access is mainly a second-order process: SDT models whether phenomenally (first-order) conscious states are accessed by reflectively (second-order) conscious processes. Consciousness and Cognition, 18(2), 561-564.

Tononi, G., \& Edelman, G. M. (1998). Consciousness and complexity. Science, 282(5395), 1846-1851. 
van Gaal, S., Ridderinkhof, K. R., Scholte, H. S., \& Lamme, V. A. (2010). Unconscious activation of the prefrontal no-go network. Journal of Neuroscience, 30(11), 4143-4150.

van Gaal, S., Lamme, V. A., Fahrenfort, J. J., \& Ridderinkhof, K. R. (2011). Dissociable brain mechanisms underlying the conscious and unconscious control of behavior. Journal of Cognitive Neuroscience, 23(1), 91-105.

Selst, M. V., \& Jolicoeur, P. (1994). A solution to the effect of sample size on outlier elimination. The Quarterly Journal of Experimental Psychology, 47(3), 631-650.

Welch, B. L. (1947). The generalization of 'Students' problem when several different population variances are involved. Biometrika, 34, 28-35.

Westbrook, A., \& Braver, T. S. (2016). Dopamine does double duty in motivating cognitive effort. Neuron, 89(4), 695-710.

Westbrook, A., \& Braver, T. S. (2015). Cognitive effort: a neuroeconomic approach. Cognitive, Affective, \& Behavioral Neuroscience, 15(2), 395-415.

Westbrook, A., Kester, D., \& Braver, T. S. (2013). What is the subjective cost of cognitive effort? Load, trait, and aging effects revealed by economic preference. PLoS ONE, 8, e68210.

Yeung, N., \& Summerfield, C. (2012). Metacognition in human decision-making: confidence and error monitoring. Philosophical Transactions of the Royal Society $B, 367(1594), 1310-1321$. 


\section{Footnotes}

1. The original DST program used in Experiment 3 of Kool et al. (2010) was provided by the first author.

2. Moderate evidence in favor of the null hypothesis was demonstrated such that high demand location had no effect on free-choice selections, $\mathrm{BF}_{\mathrm{NULL}}=3.63$, $t(195.7)=1.11, p=.26, d=.16,95$ CI $[-.12,44]$.

3. Additionally, a sensitivity analyses was performed by utilizing the two other prior scales (i.e., 1 "Wide" and $\sqrt{2}$ "Ultrawide) provided by the BayesFactor package. Coinciding with the BF associated with the default prior, no evidence was observed using the other two $r$ scales for an effect of incentive awareness on choices, $\mathrm{BF}_{\mathrm{ALT}}=.59$ (wide), $\mathrm{BF}_{\mathrm{ALT}}=.44$ (ultrawide), 


\begin{abstract}
Appendix A
Gambling Protocol (Post-DST)

"In this part of the study I am going to ask you a question about certain aspects of the task you just completed. You will have the opportunity to make an additional \$2 dollars by placing a wager on one of two options concerning the questions I ask."

"Before we ask you the question I am going to allow you to choose from two cards that I will shuffle. One card is a "WIN" card where you will win $\$ 2$ if selected and the other is a "NO WIN" card where you will not win the $\$ 2$ if selected. I will then ask you a question pertaining to the task. You can respond with either "YES" or "NO" to the question that is presented to you."

"You will then be asked to place your wager. You can either choose to stay with the answer you personally provided or choose the card that you selected prior to answering the question. If you choose to stick with your provided answer you will only win the $\$ 2$ bonus if your answer is correct. Similarly, if you choose the card that was selected prior to answering the question you will only win the $\$ 2$ bonus if you selected the "WIN" card."

"Only after you have made your wager for the question will any winnings be revealed." \#\#At this point ask if the participant has any questions and reiterate what you need to prior to moving on.

\#\#Shuffle the two cards in front of the participant and ask them to make a choice. Do not reveal the card yet; just place it to the side.

Question \#1: "Think back to the two options in the previous task that you selected between (these are the options that were displayed on the left and right of the screen). One of the two options had a tendency to switch between the tasks more often than the other. For example, one option may have displayed yellow digits more often than blue digits, whereas the other displayed the two at a somewhat similar rate."

"Please answer YES or NO to the following question: In the previous part of the task the option on the [left/right] tended to switch between colors more often than the option on the [left/right]?"

Circle one

YES




\section{Appendix A (cont.)}

"Now you will place your wager. Do you want to stick with your answer (reiterate their answer) that if correct you will win a $\$ 2$ bonus? Or do you want to choose the card you selected prior to answering the question with the chance to win $\$ 2$ if you selected the win card?"

Circle one

\section{Chose Their Answer Chose Card}

\#\#At this point you can reveal that the participants' wager was correct. We are paying everyone the $\$ 2$ regardless of their answer/choice of card.

Note: all text contained in quotation marks was read aloud by the research assistant to the participant. Text after the hash tags presented in italics are instructions to the research assistant only and were not read aloud to participants. The left/right statements within the switching question were counterbalanced across participants. 